\title{
Transvaginal Ultrasonographic Measurement of Lower Uterine Segment in Term Pregnant Patients with Previous Cesarean Section
}

\author{
Anitha Thomas*, Grace Rebekah, Reeta Vijayaselvi, Ruby Jose \\ Christian Medical College, Vellore, India \\ Email: ${ }^{*}$ anithomas@cmcvellore.ac.in
}

Received 15 July 2015; accepted 21 September 2015; published 24 September 2015

Copyright (C) 2015 by authors and Scientific Research Publishing Inc.

This work is licensed under the Creative Commons Attribution International License (CC BY). http://creativecommons.org/licenses/by/4.0/

(c) (i) Open Access

\begin{abstract}
Background: With an aim to reduce the rates of repeat cesarean section in women with a previous scar, prediction of scar rupture or dehiscence is important. If we could predict the risk of rupture by measuring the scar thickness closer to term, we could pursue a trial of scar safely. Aims: To evaluate the use of ultrasound measured thickness of lower uterine segment as one of the predictors of scar rupture or dehiscence in labour and establish a cut off beyond which trial of labour can be attempted safely. Methodology: 187 randomly selected pregnant women with history of one previous cesarean section in the past who satisfied the inclusion criteria, attending the outpatient clinic over a period of six months, at a tertiary level teaching institution in southern India were selected and counseled to undergo a transvaginal measurement of the scar region. These women were then followed up until delivery and the outcome of trial of scar, successful vaginal delivery, rupture or dehiscence of uterus was analysed in relation to the scar thickness and various other contributing factors. Results: 187 women with history of previous cesarean section, attending the outpatient clinic were randomly chosen to undergo trans vaginal scan at term. 52 underwent elective cesarean section and 135 went through trial of scar. The median cut-off of the lower uterine segment in this study population of 135 was $2.4 \mathrm{~mm}$. The sensitivity was $90.9 \%$, specificity was $43.5 \%$, positive predictive value was $12.5 \%$, and negative predictive value was $98.3 \%$ at this cutoff for scar rupture or dehiscence. Conclusions: The lower uterine scar thickness could be a useful tool to predict scar rupture. This could aid in making decisions regarding induction of labour with oxytocin in women with previous cesarean section.
\end{abstract}

\section{Keywords}

TOLAC, Transvaginal Ultrasonography, Scar Thickness

\footnotetext{
*Corresponding author.
}

How to cite this paper: Thomas, A., Rebekah, G., Vijayaselvi, R. and Jose, R. (2015) Transvaginal Ultrasonographic Measurement of Lower Uterine Segment in Term Pregnant Patients with Previous Cesarean Section. Open Journal of Obstetrics and Gynecology, 5, 646-653. http://dx.doi.org/10.4236/ojog.2015.511091 


\section{Introduction}

Vaginal birth after cesarean delivery is one of the most talked about controversies in modern obstetrics. Most reports in literature indicate that approximately $60 \%-80 \%$ of trials of labour after previous cesarean delivery result in a successful vaginal birth [1]. Every obstetrician is faced with the dilemma of whether to allow vaginal delivery or to perform an elective repeat cesarean section in patients with history of previous cesarean section. The major fear is allowing women to undergo trial of scar rupture $(0.1 \%-0.5 \%)$ or dehiscence $(0.5 \%-1.5 \%)$ [2]. As the outcome for both the mother and the baby in the event of rupture uterus is catastrophic, if uterine rupture or dehiscence can be predicted, trial of labor after cesarean section (TOLAC) can be managed more safely.

In most cases, the etiological factor contributing to the rupture is unknown. Several predictors and prediction model scores have been evaluated to enable the practicing physician to be able to safely recruit women for trial of labour. Various factors like prior vaginal delivery, cervical dilatation at posting, labor induction, body mass index and maternal medical conditions have been studied and have not been able to successfully develop a woman specific predictor model for successful Vaginal Birth after Cesarean section (VBAC) or rupture uterus [3] [4]. Ultrasonographic evaluation of the lower uterine scar and its usefulness in prediction of rupture uterus during labor had been studied in the past [5]-[8]. The applicability of trans vaginal ultrasound evaluation of lower uterine segment thickness as a part of the predictive models has only been cited recently in one article which also studies the other parameters contributing to rupture uterus [9].

Our institution is one of the few centers in our country where trial of labour following previous cesarean delivery is being allowed. 70 - 80 women with history of previous cesarean scar are admitted into labor ward every month. Of these $60 \%$ - 65\% undergo trial of labour. The remaining 35\% - 40\% of them undergo elective repeat cesarean section for indications such as CPD, macrosomia, placenta praevia, malpresentation and of late at patients request. Trial of scar is attempted in about $55 \%$ with a VBAC rate of about $50 \%$. The incidence of scar rupture and dehiscence as per our earlier institutional data which used to be $4 \%-8 \%$, has reduced to almost less than $1 \%$ as the decision for cesarean section is now taken earlier with the evolving practice guidelines. In an effort to improve the TOLAC rates and reduce the fear of rupture uterus, the various factors predisposing to uterine rupture and the probability of predicting the rupture were looked into.

The aim of this study was to evaluate the use of ultrasound measured thickness of lower uterine segment as one of the predictors of scar rupture/dehiscence in labour. Based on the above observation, we aimed to establish a cut-off value of lower segment thickness above which vaginal birth after cesarean section could be attempted safely at our institution. We also aimed at studying the role of ultrasound evaluation as a part of the predictor model along with other variables, for success of VBAC or rupture uterus.

\section{Methods \& Materials}

A prospective study was conducted on 187 randomly selected pregnant women with history of one previous cesarean section in the past, attending the outpatient clinic over a period of six months, at a tertiary level teaching institution in Southern India. The inclusion criteria were pregnant women with history of one previous caesarean section of gestational age more than 37 weeks with singleton pregnancy in cephalic presentation, intact membranes and not in labour. We excluded women with an abnormal presentation, active labour and those with ruptured membranes. Informed consent was obtained. Transvaginal ultrasonographic measurement of the Lower Uterine Segment (LUS) was performed on an empty bladder, once at any time between 37 \& 41 weeks. All these scans were done mostly by a single investigator. These women were allowed to either undergo trial of labour or underwent repeat elective cesarean section according to the usual protocol followed by the treating unit. The attending obstetrician was unaware of the ultrasound findings.

The measurement was taken using TOSHIBA Model SSA-320A machine with a $6 \mathrm{MHz}$ vaginal probe, within 30 minutes of voiding. 3 measurements were taken and an average of the 3 was taken as the final LUS thickness.

Sonographically, the lower uterine segment has a three layered structure. The outer layer is the visceral parietal peritoneal reflection, which is juxtaposed to the muscularis layer of the bladder. The middle layer is the myometrium. The innermost layer is the decasualized endometrial layer outer to the chorioamniotic membrane (Figure 1). Only the muscle layer was measured at its thinnest portion. The measurements were repeated three times and a mean was obtained.

In most cases the vaginal probe could not visualize the actual scar tissue but the area 1 - $2 \mathrm{~cm}$ caudal to cesarean scar. 


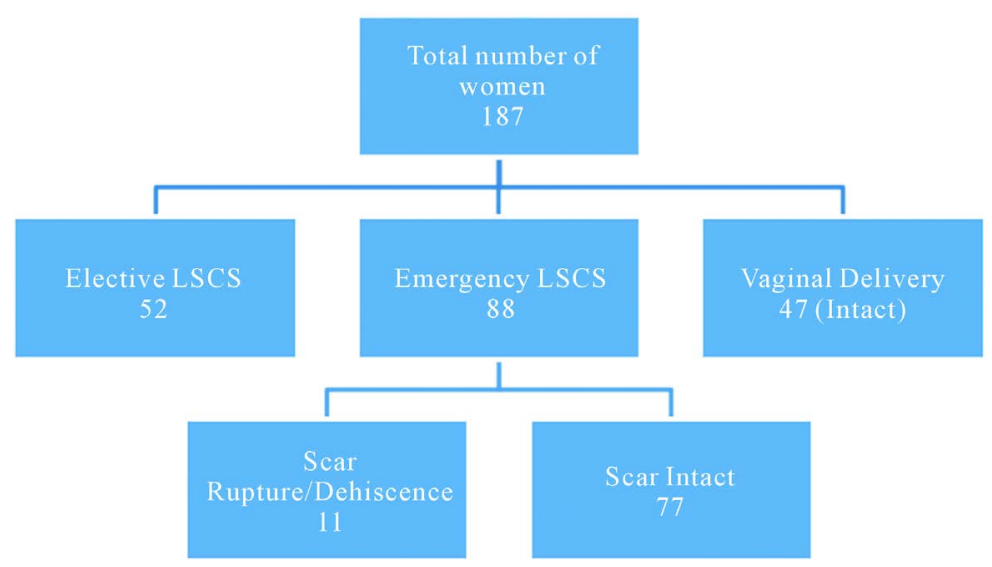

Figure 1. Algorithm showing the distribution of patients and their delivery outcome.

The patients were then followed up after delivery and the outcome of the delivery was documented. Demographic data, history of their previous section, the LUS thickness, Gestational age, nature of labor, use of pitocin, meconium staining of hind waters, pre-labour rupture of membranes (PROM), birth weight and Apgar of baby, complications and other details were documented. The presence or absences of uterine scar dehiscence or rupture was noted after delivery. Uterine dehiscence was defined as separation of the muscular layer with an intact serosa. The mere thinning of the scar was not classified as dehiscence. In patients who delivered vaginally the absence of profuse vaginal bleeding or shock was considered equivalent to an intact uterine scar.

The study group was divided into 2 groups based on their scar integrity following delivery. Those who underwent Elective cesarean section were excluded from the analysis. The baseline characteristics were analyzed. Univariate and Bivariate analysis was done on various factors like age, occupation, gestational age at delivery, obstetric parity, indication for previous cesarean section, mode of labor, LUS thickness, duration of labor, use of pitocin and birth weight of baby.

Continuous data was analyzed using students t test and chi square test was used for the categorical data. Various risk factors were looked into and risk factor analysis was done using logistic regression. The risk factors that were studied were maternal age, weight, gestational age at delivery, LUS thickness, mode of onset of labor, use of pitocin, baby weight, indication for previous cesarean section and parity. Univariate followed by multivariate analysis was done on the above risk factors and adjusted for the effects of each other.

ROC curve was constructed for the various risk factors and the area under curve obtained for the significant factors. The median cut off was obtained and the sensitivity, specificity, positive and negative predictive value of LUS in prediction of scar dehiscence/ rupture was calculated.

\section{Results}

187 women with history of previous cesarean section attending the outpatient clinic were randomly chosen to undergo trans vaginal scan at term. 52 underwent elective cesarean section and 135 went through trial of scar.

The baseline characteristics of these patients were as outlined in the Table 1 . The two groups were not significantly different with regards to the gestational age at delivery, occupation, obstetric score and indication for previous cesarean cesarean section. The thickness of the LUS was significantly different ( $p$ value of 0.028 ) between the two groups with the scar rupture group tending to have thinner scars than the group with the intact scar. With regards to mode and duration of labor the groups were similar and pitocin seemed to be used more in the rupture group ( $\mathrm{p}$ value of 0.001 ) With respect to decelerations in labor, meconium stained amniotic fluid and intra op complications the two groups were similar. There were a total of 17 cases with PROM.

Labour outcomes (Table 2) of the two groups resulted in 11 patients with scar rupture/dehiscence and 124 with intact scars. 47 of the 135 women who underwent TOLAC had a successful vaginal birth with a VBAC rate of $34.8 \%$. Serious intraoperative complications like postpartum haemorrhage were significantly higher in patients with rupture of the scar (45\% versus $0.8 \%$ ). The neonatal outcomes of APGAR $<7$ were found in 2 out of the 11 scar rupture events 
Table 1. Descriptive statistics.

\begin{tabular}{|c|c|c|c|c|c|}
\hline & \multicolumn{2}{|c|}{$\begin{array}{c}\text { Cases (11) } \\
\text { Scar Rupture/Dehiscence }\end{array}$} & \multicolumn{2}{|c|}{$\begin{array}{l}\text { Controls (124) } \\
\text { Scar Intact }\end{array}$} & \multirow{2}{*}{ p value } \\
\hline & $\mathbf{n}$ & $\%$ & $\mathbf{n}$ & $\%$ & \\
\hline Maternal Age & \multicolumn{2}{|l|}{$27.5 \pm 4.4$} & \multicolumn{2}{|c|}{$25.8 \pm 4.5$} & 0.274 \\
\hline Gestational Age at delivery & \multicolumn{2}{|l|}{$38.4 \pm 1.3$} & \multicolumn{2}{|c|}{$38.4 \pm 1.2$} & 0.452 \\
\hline Previous LSCS & & & & & 0.697 \\
\hline Elective & 3 & 27.3 & 25 & 20.2 & \\
\hline Emergency & 8 & 72.7 & 99 & 79.8 & \\
\hline \multicolumn{6}{|l|}{ LUS thickness (mm) } \\
\hline$\leq 2.4$ & \multicolumn{2}{|l|}{$10(90.9)$} & \multicolumn{2}{|c|}{$70(56.5)$} & $0.028 *$ \\
\hline$>2.4$ & \multicolumn{2}{|l|}{$1(9.1)$} & \multicolumn{2}{|c|}{$54(43.5)$} & \\
\hline \multicolumn{6}{|l|}{ Mode of Labor } \\
\hline Spontaneous & 5 & 45.5 & 90 & 72.6 & 0.083 \\
\hline Induced & 6 & 54.5 & 34 & 27.4 & \\
\hline \multicolumn{6}{|l|}{ Pitocin } \\
\hline Yes & 9 & 81.8 & 38 & 30.9 & $0.001^{*}$ \\
\hline No & 2 & 18.2 & 85 & 69.1 & \\
\hline
\end{tabular}

Table 2. Labour outcomes of Trial of Scar.

\begin{tabular}{|c|c|c|c|c|c|}
\hline & \multicolumn{2}{|c|}{ Cases (11) } & \multicolumn{2}{|c|}{ Controls (124) } & \\
\hline & $\mathbf{n}$ & $\%$ & $\mathbf{n}$ & $\%$ & \\
\hline \multicolumn{6}{|l|}{ Delivery } \\
\hline LSCS & 11 & 100.0 & 77 & 62.1 & \multirow{4}{*}{0.137} \\
\hline Vaginal Normal & - & - & 22 & 17.7 & \\
\hline Vaginal Forceps & - & - & 19 & 15.3 & \\
\hline Vaginal Suction & - & - & 6 & 4.8 & \\
\hline \multicolumn{6}{|l|}{ Delivery outcome } \\
\hline Normal Delivery & - & - & 47 & 37.9 & \multirow[t]{2}{*}{$0.008^{*}$} \\
\hline Emergency LSCS & 11 & 100.0 & 77 & 62.1 & \\
\hline \multicolumn{6}{|l|}{ Intraop Complication } \\
\hline Bladder Injury & - & 45.5 & 1 & 0.8 & \multirow{3}{*}{$0.048^{*}$} \\
\hline $\mathrm{PPH}$ & 5 & & 1 & 0.8 & \\
\hline Adhesions & & & 2 & 1.6 & \\
\hline Birthweight (Grams) & \multicolumn{2}{|c|}{$3189.1 \pm 313.9$} & \multicolumn{2}{|c|}{$3162.3 \pm 397.8$} & 0.808 \\
\hline$\leq 3160$ & \multicolumn{2}{|c|}{$4(36.4)$} & \multicolumn{2}{|c|}{$62(51.7)$} & \\
\hline$>3160$ & \multicolumn{2}{|c|}{7 (63.6) } & \multicolumn{2}{|c|}{$58(48.3)$} & 0.331 \\
\hline \multicolumn{6}{|l|}{ Apgar at 5 minutes } \\
\hline$\leq 7$ & 2 & 18.2 & - & - & \multirow[t]{2}{*}{$0.006^{*}$} \\
\hline$>7$ & 9 & 81.8 & 122 & 100.0 & \\
\hline
\end{tabular}


Risk factor analysis (Table 3) was done using Logistic regression against cases and controls. Bivariate analysis (unadjusted) was carried out for the following variables namely, maternal age, maternal weight, gestational age, indication for previous cesarean, LUS thickness, mode of onset of labor use of pitocin and birthweight. When the significance was fixed at $25 \%$ the only variables which were significant were pitocin use, LUS thickness, and mode of onset of labor. In the multivariate analysis adjusting for effect of the use of pitocin, LUS thickness and Mode of labor, only pitocin use was significant at 5\% with OR of 9.27. Use of pitocin still had an influence on the rate of rupture even after adjusting for the mode of onset of labor and LUS thickness. The median cut off of the lower uterine segment in this study population was $2.4 \mathrm{~mm}$. The area under curve for the ROC constructed for these risk factors with a cut off for LUS thickness fixed at $2.4 \mathrm{~mm}$ as increased risk, was $0.806(665-0.94) \mathrm{p}$ value of 0.001 . The sensitivity was $90.9 \%$, specificity was $43.5 \%$, positive predictive value was $12.5 \%$, and negative predictive value was $98.3 \%$ at this cut off (Figure 2, Figure 3).

\section{Discussion}

Trial of Labour after cesarean section has been a subject of much debate and discussion in the recent past. Various researchers have looked into factors which may affect the outcome of the labor. Previous normal vaginal delivery, spontaneous labor, non recurring indication for previous caesar are some of the favourable factors whereas induced labor, short interdelivery interval, infection during peripartum period in the previous pregnancy are factors which would result in poorer outcome. Trans vaginal scan in patients with previous scar to measure the lower uterine segment thickness has been evaluated as one such factor which could influence the outcome of labor in women with previous cesarean section. It has been evaluated as a predictor tool for rupture/dehiscence

Table 3. Risk factor analysis of predisposing factors in rupture of scar.

\begin{tabular}{|c|c|c|c|c|c|c|c|c|c|c|}
\hline \multirow{3}{*}{ Risk Factors } & \multicolumn{4}{|c|}{ Scar } & \multicolumn{3}{|c|}{ Unadjusted } & \multicolumn{3}{|c|}{ Adjusted } \\
\hline & \multicolumn{2}{|c|}{ Rupture } & \multicolumn{2}{|c|}{ Intact } & \multirow{2}{*}{ OR } & \multirow{2}{*}{$95 \%$ CI } & \multirow{2}{*}{$P$ value } & \multirow{2}{*}{ OR } & \multirow{2}{*}{$95 \% \mathrm{CI}$} & \multirow{2}{*}{ p value } \\
\hline & n & $\%$ & $\mathbf{n}$ & $\%$ & & & & & & \\
\hline $\begin{array}{l}\text { Maternal Age } \\
\text { (Wks) }\end{array}$ & \multicolumn{2}{|c|}{$27.45 \pm 4.3$} & \multicolumn{2}{|c|}{$25.8 \pm 4.5$} & 1.07 & $0.95-1.22$ & 0.275 & - & - & - \\
\hline $\begin{array}{l}\text { Maternal Weight } \\
(\mathrm{Kg})\end{array}$ & \multicolumn{2}{|c|}{$60.0 \pm 13.8$} & \multicolumn{2}{|c|}{$60.0 \pm 10.6$} & 1.00 & $0.94-1.07$ & 0.994 & - & - & - \\
\hline $\begin{array}{l}\text { Gestational Age } \\
\text { (Wks) }\end{array}$ & \multicolumn{2}{|c|}{$39.7 \pm 1.4$} & \multicolumn{2}{|c|}{$39.5 \pm 1.1$} & 1.25 & $0.69-2.25$ & 0.449 & - & - & - \\
\hline \multicolumn{11}{|l|}{ Previous LSCS } \\
\hline Elective (R) & 3 & 27.3 & 25 & 20.2 & 1 & - & - & - & - & - \\
\hline Emergency & 8 & 72.7 & 99 & 79.8 & 0.67 & & & & & \\
\hline \multicolumn{11}{|l|}{ LUS (mm) } \\
\hline$\leq 2.4$ & 10 & 90.9 & 70 & 56.5 & 0.13 & & & 6.65 & $0.78-56.6$ & 0.08 \\
\hline$>2.4(\mathrm{R})$ & 1 & 9.1 & 54 & 43.5 & 1 & $0.02-1.04$ & 0.055 & 1 & - & - \\
\hline \multicolumn{11}{|l|}{ Mode of Labor } \\
\hline Spontaneous (R) & 5 & 45.5 & 90 & 72.6 & 1 & - & - & 1 & - & - \\
\hline Induced & 6 & 54.5 & 34 & 27.4 & 3.2 & $0.9-11.1$ & 0.070 & 1.22 & $0.27-5.5$ & 0.789 \\
\hline \multicolumn{11}{|l|}{ Pitocin } \\
\hline Yes & 9 & 81.8 & 38 & 30.9 & 10.06 & $2.07-48.83$ & $0.004 *$ & 8.08 & $1.3-49.0$ & $0.023^{*}$ \\
\hline No (R) & 2 & 18.2 & 85 & 69.1 & 1 & - & - & 1 & - & - \\
\hline \multicolumn{11}{|l|}{ Birthweight (Grams) } \\
\hline$\leq 3160(\mathrm{R})$ & 4 & 36.4 & 62 & 51.7 & 1 & - & - & - & - & - \\
\hline$>3160$ & 7 & 63.6 & 58 & 48.3 & 1.87 & $0.52-6.73$ & 0.337 & & & \\
\hline
\end{tabular}




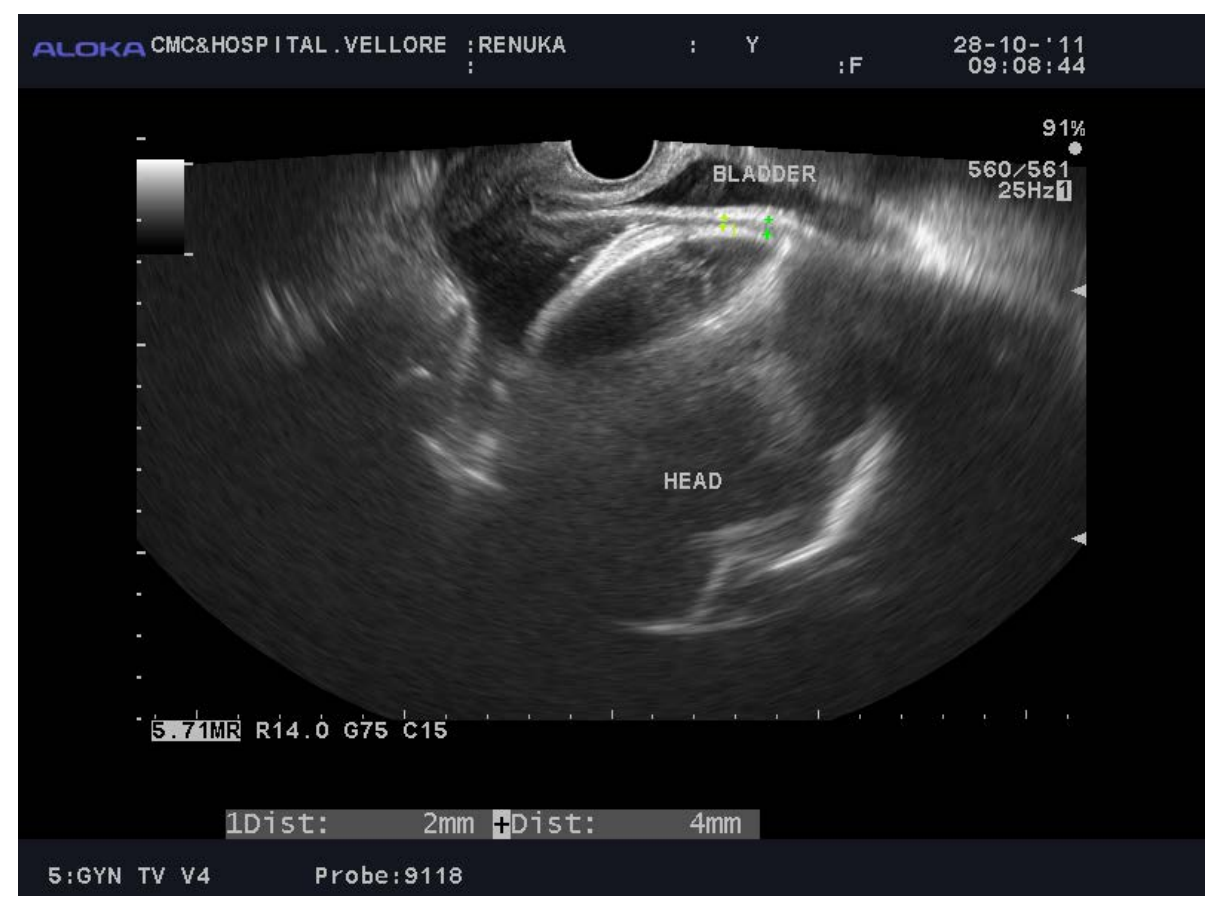

Figure 2. Image showing the position of the lower uterine segment with the muscle portion shown as the dark portion between the two bright lines, the bladder and the fetal head.

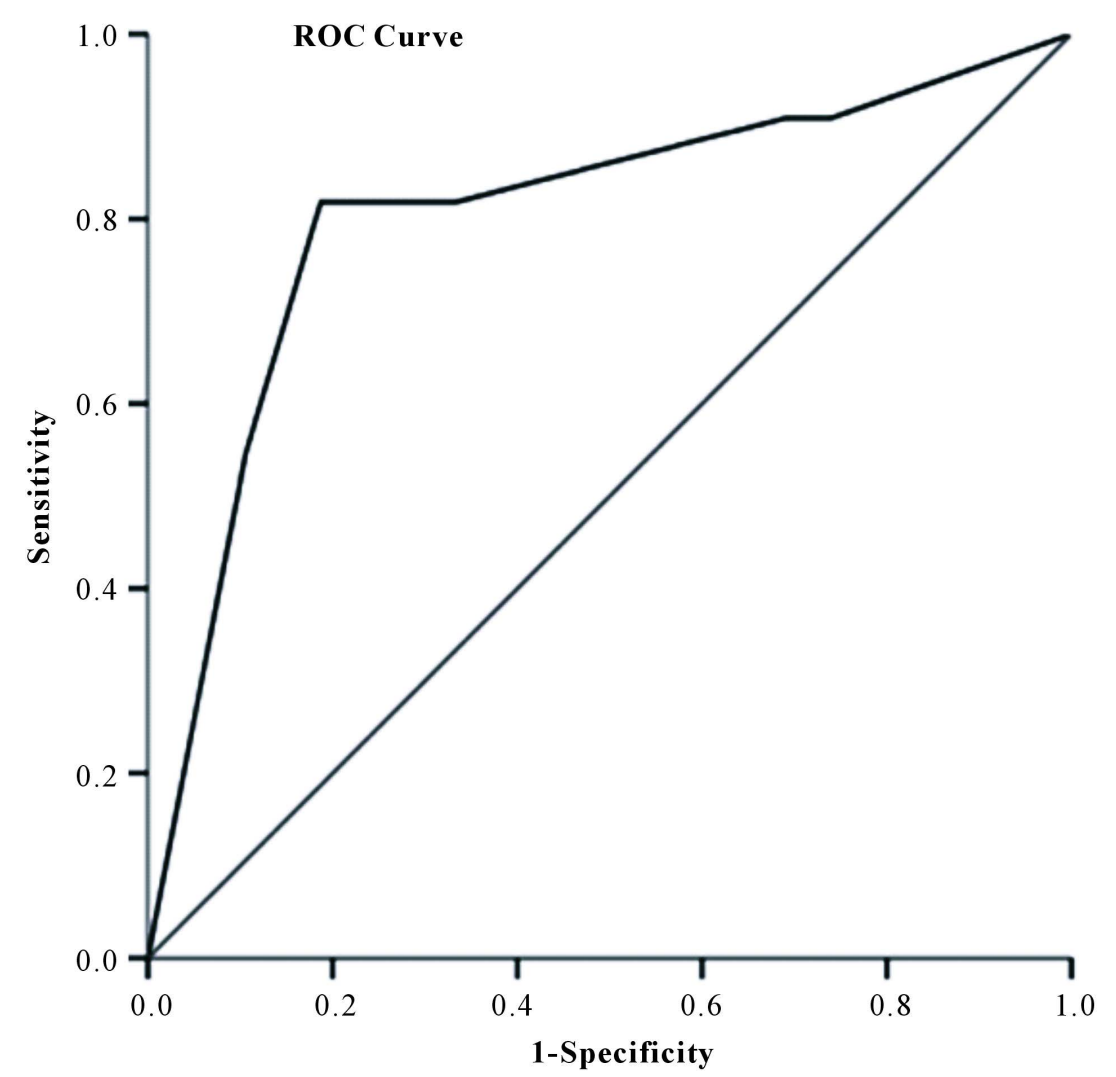

Diagonal segments are produced by ties.

Figure 3. ROC curve of the measured values of the LUS thickness of the study population. Area under the curve $=0.806(0.65$ to 0.96$)$, $\mathrm{p}$ value $=0.001$. 
in some studies [5] [8]. Other investigators have compared the transabdominal measurement on full bladder versus the transvaginal measurement on empty bladder to assess the risk of rupture uterus [7] [10]. Irrespective of whether transabdominal or tranvaginalr route, the measured thickness at a particular cut-off value had a high negative predictive value in predicting scar rupture. Thus the patients with thick lower uterine scar are less likely to have a scar dehiscence/rupture. In our series of patients, the negative predictive value was $98.2 \%$ at a cut off of $2.4 \mathrm{~mm}$.

As the test is not efficient in itself as screening tool its use was further evaluated as a part of a predictor model. Various predictor models have been assessed in the past for screening patients for trial of scar in previous cesarean section. Though many of them assess known risk factors such as previous vaginal delivery, parity, inter pregnancy interval etc. none of them could positively predict rupture during trial of scar. One of these studies have also assessed the risk incorporating the lower uterine scar thickness in the model and found that along with thickness the other factors which were strongly associated with rupture were the single layer closure and inter delivery interval $<18$ months [11]. A recently concluded metanalysis has established support for the use of antenatal LUS measurements in the prediction of a uterine defect during trial of labour [12].

When we assessed various factors which affected the outcome in our series of patients, the risk factors which stood out as significant were the use of pitocin, mode of onset of labor and the lower uterine segment thickness at the univariate level. When adjusted for each other, only use of pitocin proved to be almost 8 times more risk. In patients where labor has to be induced, a thin lower segment could mean more chances of rupture. If the thickness of the segment was $<2.4 \mathrm{~mm}$, the risk of rupture was about 6.5 times more with use of pitocin.

The limitations of our study were the small numbers of patients and that we did not include some other risk factors like inter delivery interval in our risk factor analysis.

\section{Conclusion}

In summary, though prediction of scar rupture in a case of previous cesarean section could be difficult, the decision making regarding the mode of delivery could be aided by measuring the lower uterine segment thickness whereby the women with lower uterine segment thickness $<2.4 \mathrm{~mm}$ could be counseled against induction of labor. In women with spontaneous onset of labor, the LUS thickness could be useful in predicting those who were unlikely to have scar rupture/dehiscence.

\section{Conflict of Interest}

There are no conflicts of interest in this study.

\section{References}

[1] Grobman, W.A. (2010) Rates and Prediction of Successful Vaginal Birth after Cesarean. Seminars in Perinatology, 34, 244-248. http://dx.doi.org/10.1053/j.semperi.2010.03.003

[2] Rosen, M.G., Dickinson, J.C. and Westhoff, C.L. (1991) Vaginal Birth after Cesarean: A Meta-Analysis of Morbidity and Mortality. Obstetrics \& Gynecology, 77, 465-470.

[3] Macones, G.A., Cahill, A.G., Stamilio, D.M, Odibo, A., Peipert, J. and Stevens, E.J. (2006) Can Uterine Rupture in Patients Attempting Vaginal Birth after Cesarean Delivery Be Predicted? American Journal of Obstetrics \& Gynecology, 195, 1148-1152. http://dx.doi.org/10.1016/j.ajog.2006.06.042

[4] Grobman, W.A., Lai, Y., Landon, M.B., Spong, C.Y., Leveno, K.J., Rouse, D.J., et al. (2008) Prediction of Uterine Rupture Associated with Attempted Vaginal Birth after Cesarean Delivery. American Journal of Obstetrics \& Gynecology, 199, 30.e1-e5. http://dx.doi.org/10.1016/j.ajog.2008.03.039

[5] Asakura, H., Nakai, A., Ishikawa, G., Suzuki, S. and Araki, T. (2000) Prediction of Uterine Dehiscence by Measuring Lower Uterine Segment Thickness Prior to the Onset of Labor: Evaluation by Transvaginal Ultrasonography. Journal of Nippon Medical School, 67, 352-356.

[6] Bergeron, M.-E., Jastrow, N., Brassard, N., Paris, G. and Bujold, E. (2009) Sonography of Lower Uterine Segment Thickness and Prediction of Uterine Rupture. Obstetrics \& Gynecology, 113, 520-522. http://dx.doi.org/10.1097/AOG.0b013e31818c15a7

[7] Rozenberg, P., Goffinet, F., Phillippe, H.J. and Nisand, I. (1996) Ultrasonographic Measurement of Lower Uterine Segment to Assess Risk of Defects of Scarred Uterus. The Lancet, 347, 281-284. http://dx.doi.org/10.1016/S0140-6736(96)90464-X 
[8] Qureshi, B., Inafuku, K., Oshima, K., Masamoto, H. and Kanazawa, K. (1997) Ultrasonographic Evaluation of Lower Uterine Segment to Predict the Integrity and Quality of Cesarean Scar during Pregnancy: A Prospective Study. The Tohoku Journal of Experimental Medicine, 183, 55-65.

[9] Jeanne-Marie Guise, K.E. (2010) Detailed Evaluation of Screening Tools for Predicting Vaginal Birth After Cesarean. http://www.ncbi.nlm.nih.gov/books/NBK44570/

[10] Marasinghe, J.P., Senanayake, H., Randeniya, C., Seneviratne, H.R., Arambepola, C. and Devlieger, R. (2009) Comparison of Transabdominal Versus Transvaginal Ultrasound to Measure Thickness of the Lower Uterine Segment at Term. International Journal of Gynecology \& Obstetrics, 107, 140-142. http://dx.doi.org/10.1016/j.ijgo.2009.05.022

[11] Bujold, E., Jastrow, N., Simoneau, J., Brunet, S. and Gauthier, R.J. (2009) Prediction of Complete Uterine Rupture by Sonographic Evaluation of the Lower Uterine Segment. American Journal of Obstetrics \& Gynecology, 201, 320.e1-e6. http://dx.doi.org/10.1016/j.ajog.2009.06.014

[12] Kok, N., Wiersma, I.C., Opmeer, B.C., de Graaf, I.M., Mol, B.W. and Pajkrt, E. (2013) Sonographic Measurement of Lower Uterine Segment Thickness to Predict Uterine Rupture during a Trial of Labor in Women with Previous Cesarean Section: A Meta-Analysis. Ultrasound in Obstetrics \& Gynecology, 42, 132-139. http://dx.doi.org/10.1002/uog.12479 